\title{
Early Postnatal Valproic Acid Exposure Increase the Protein Level of Astrocyte Markers in Frontal Cortex of Rat
}

\author{
Tamanna Jahan Mony ${ }^{1}$, Jae Won Lee ${ }^{1,2}$, Sung Soo Kim ${ }^{1}$, Wanjoo Chun ${ }^{1}$, Hee Jae Lee ${ }^{1}$ \\ ${ }^{1}$ Department of Pharmacology, Kangwon National University School of Medicine, Chuncheon, ${ }^{2}$ National Medicine Research Center, Korea \\ Research Institute of Bioscience and Biotechnology, Chungju, Korea
}

\begin{abstract}
Objective: In our previous study, it has been reported that valproic acid (VPA) effects gliogenesis and increases the number of glial precursor cells during the early postnatal period. However there is no specific report that whether this process is going on up to the age of mature brain development and the consequence effect of this ongoing gliogenesis process.

Methods: As an ongoing study, using Immunoblotting analysis, we checked the level of glial protein and glial-derived factor markers in the frontal cortex of a rat brain at postnatal day (PND) 21.

Results: The finding of the study suggests that, in the VPA group $(p<0.05)$, early exposure elicited significantly to increase the expression level of glial protein cells at PND 21 in the frontal cortex of rat brain.

Conclusion: Therefore we suggest that, alter gliogenesis and abnormal number of glial cells modulate the neurobiological dysfunction and induces the risk of neurodevelopmental disorders.
\end{abstract}

KEY WORDS: Postnatal development; Valproic acid; Immunoblotting; Neuroglia; Frontal cortex.

\section{INTRODUCTION}

The largest set of glia namely astrocyte is imperative for the development of the nervous system, synaptic plasticity, and synaptogenesis process. Astrocyte dysfunction may lead to the aberration of neuronal circuitry that underlies for different neurodevelopmental disorders. The reasonable proportion of neuron and glia is essential for mature brain function. ${ }^{1)}$ An increased number of astrocyte in the frontal cortex can modify the establishment of major neuron-glial circuit formation. ${ }^{2)}$ A surplus astrocyte during glial-neural circuit formation could be another locus for pathogenetic factors of neuropsychiatric disorders like autism spectrum disorder (ASD). Unlike neuron, glial cell retains their ability to proliferate in most postnatal

Received: February 2, 2017 / Revised: March 5, 2017

Accepted: March 29, 2017

Address for correspondence: Hee Jae Lee, PhD

Department of Pharmacology, Kangwon National University

School of Medicine, 1 Kangwondaehak-gil, Chuncheon 24341,

Korea

Tel: +82-33-2508850, Fax: +82-33-2595635

E-mail: heejaelee@kangwon.ac.kr

ORCID: https://orcid.org/0000-0002-7816-6444 brain areas and adult subject. ${ }^{3,4)}$ Early valproic acid (VPA) exposure leads to increased precursor proliferation at postnatal day (PND) 4. ${ }^{5)}$ The finding of the study shown that VPA-induced mitotic stimulation increased the cells number and survived long-term up to PND 21. According to our previous data, we found that early VPA exposure leads to increase the number of astrocyte by PND 21. This means that VPA stimulates gliogenesis during the developmental stage and this altered gliogenesis modulates behavioral changes of a rat. ${ }^{2,5)}$ As a continuation of these works, we analyzed the expression of the protein level of astrocyte markers. The expression of increased level of astrocyte marker indicates the much more production of astrocyte in the frontal cortex. That means the process of gliogenesis is going on. These abundant number of astrocytes effect the maintenance of normal glia and neuron ratio and the associated normal function in the frontal cortex area. Moreover, in different human studies reported that the expression of increased level of astrocyte marker like glial fibrillary acidic protein (GFAP) and major component protein of astrocytic gap junction were increased in the superior frontal cortex of post mortem brain tissues of ASD patient. ${ }^{6,7)}$ Many recent studies implicated that glia

(ㄷ) This is an Open-Access article distributed under the terms of the Creative Commons Attribution Non-Commercial License (http://creativecommons.org/licenses/by-nc/4.0) which permits unrestricted non-commercial use, distribution, and reproduction in any medium, provided the original work is properly cited. 
especially the astrocytes have a pivotal role in synapnse formation and function. ${ }^{8-10)}$ Altered gliogenesis or defect in astrocyte function during early development may contribute to the progression of different neurodevelopmental disorders such as autism, schizophrenia and fragile $X$ syndrome. ${ }^{8-12)}$ We assumed that analysis of expression level of glial cell would provide evidence of amendment of gliogenesis in adult brain region which might be a new target for new therapeutic intervention or future experimental approaches for the neurodevelopmental disorders that characterized by the defects in synapse formation and function.

\section{METHODS}

\section{Animal Treatment}

We obtained a pregnant Sprague-Dawley rat from Taconic (Seongnam, Korea) and accommodated it with a standard light-dark cycle (12 hours), ambient temperature $\left(22 \pm 2{ }^{\circ} \mathrm{C}\right)$ and humidity $(55 \pm 5 \%)$, with liberal access to pellet and water. We checked the health of the rat every day. The newborn pups were born on a day considered as PND 0. On PND 2 to 4, the rat pups were weighed and injected with $300 \mathrm{mg} / \mathrm{kg}$ of valproic acid sodium (SigmaAldrich, St. Louis, MO, USA) at the dorsal neck region. All animal treatments including anesthesia, euthanasia, and administration of drug were carried out following the principles of Laboratory Animal Care (NIH publication N0-85-23, revised 1985) and the Korean Academy of Medical Sciences.

\section{Immunoblotting Analysis}

We collected frontal brain tissue at PND 21. Frontal cortex tissues were homogenized using a protein extraction solution (Pro-prep ${ }^{\mathrm{TM}}$; iNtRON Biotechnology, Seongnam, Korea). The lysates were then centrifuged at $14,000 \mathrm{rpm}$ and the supernatant was collected for storage. Protein of 5-10 $\mu \mathrm{g}$ was denatured and resolved using $8-12 \%$ SDS-PAGE, then transferred to a nitrocellulose membrane. We blocked the membranes with 5\% skim milk in TBST or PBST containing $0.05 \%$ to $0.2 \%$ Tween 20 for 1 hour. The membranes were incubated with the primary antibodies GFAP (1:1,000; Cell Signaling, Danvers, MA, USA), glial-derived neurotrophic factor (GDNF, 1:1,000; Santa Cruz Biotechnology, Santa Cruz, CA, USA) and excitatory amino acid transporter (EAAT1, 1:1,000; Abcam, Cambridge, UK) overnight at $4^{\circ} \mathrm{C}$. After that, the membranes were incubated with a peroxidase-conjugated secondary antibody $(1: 2,500)$ for 2 hours at room temperature. Specific bands were detected using the ECL system and were analyzed using Image J software (http://imagej.nih.gov/ij/download/) for quantifi-
A

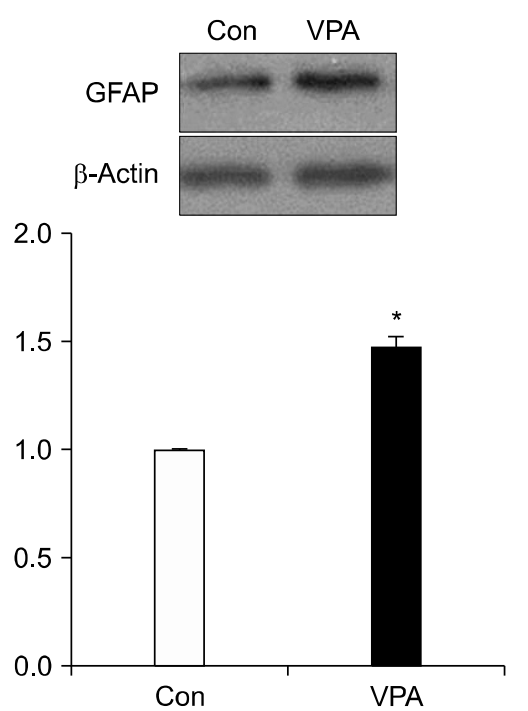

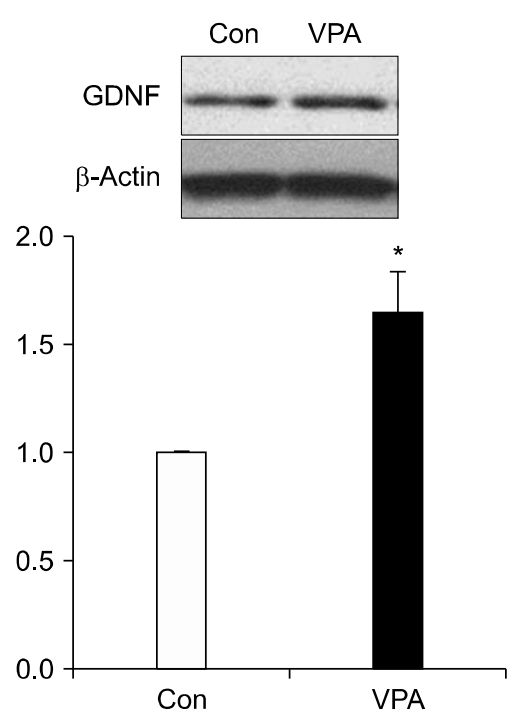

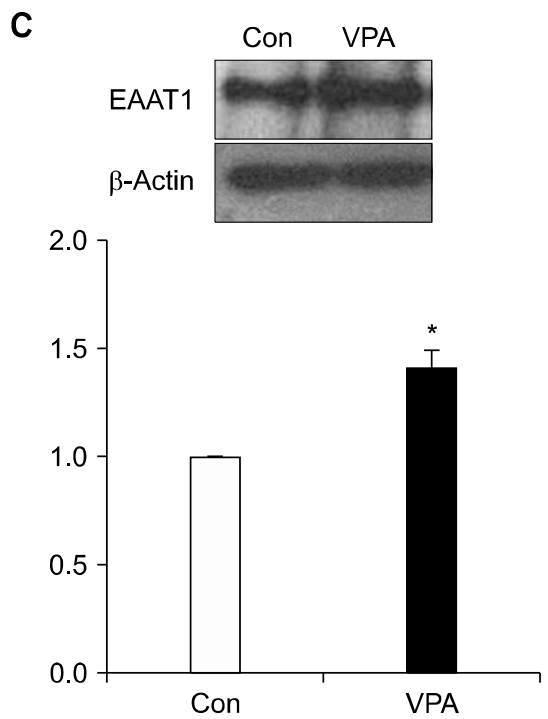

Fig. 1. Immunoblotting of glial cell and cell-derived factors protein in the frontal cortex at postnatal day 21. Valproic acid (VPA) treated rats significantly increased glial cell marker protein compared with controls (Con; $n=4 /$ group). Data were presented as mean \pm standard error of mean. GFAP, glial fibrillary acidic protein; GDNF, glial cell-derived neurotrophic factor; EAAT1, excitatory amino acid transporter. ${ }^{*} p<0.05$. 
cation purposes. $\beta$-actin was used as a loading control and the Immunoblotting intensity was normalized with $\beta$-actin immunoreactivity.

\section{Statistical Analysis}

IBM SPSS Statistics ver. 20.0 (IBM Co., Armonk, NY, USA) was used. All results were expressed as the mean \pm standard error of mean for each group. We used the Student's $t$ test $(p<0.05)$ to determine the statistical differences between the two groups.

\section{RESULTS}

Using the immunoblotting analysis, the expression of the protein levels of the different glial cells or glial derivative factors were found to increase in the cortical brain tissue at PND 21. We checked the protein levels of three astrocyte markers, considering their structural and functional importance. We found that, in VPA-treated rats, the astrocyte marker proteins (GFAP, GDNF, and EAAT1) were increased in the adolescent cortex (Fig. 1). Lee et al. ${ }^{5)}$ already shown in 2015 that VPA elicited the proliferation of astrocyte precursor both in vitro and in vivo. We assume that increasing the number of astrocyte precursor cells in the juvenile cortex during the early postnatal period affects the number of astrocytes in the adult cortex. We analyzed the Student's $t$ test $(p<0.05)$ to measure the amount of increase of protein expression of both the VPA and control groups.

\section{DISCUSSION}

The increased protein levels indicated that there were an excess number of astrocyte cells due to the alteration of gliogenesis in the adolescent rat cortex. In our previous study we reported that early postnatal VPA exposure may lead to neurodevelopmental deficits and induce autistic-like behaviors. ${ }^{2}$ The finding of this study supports our previous hypothesis that during glial-neural circuit formation, the surplus number of astrocytes disrupts the normal formation process and also affects functional deficits like synapse formation, maturation function and the elimination process. ${ }^{2,7-10,13,14)}$ Moreover, the surplus number of astrocytes may inhibit the formation of GABAergic synapses and glutamatergic differentiation. ${ }^{15,16)}$ According to Lee and co-workers, ${ }^{5)}$ it supported that early postnatal exposure inhibits histone deacetylase, which is related to altered gliogenesis. The results indicated that VPA affects glial cell proliferation at the critical time of progenitor cell division. Eventually, the increased number of astrocytes in the juvenile rat frontal cortex further presented as a potential cause of social and behavioral deficits in the rat. ${ }^{2,5)}$ Furthermore, there is a possibility that an abnormal increase of astrocyte cells in the postnatal brain might be able to produce excess signal molecules, affecting the excitatory/inhibitory (E/I) imbalance in postnatal VPA-treated rats. In some animal models, alteration in E/I balances within the cortex were found to decrease social motivation and create a social and behavioral deficit as is the case with ASD. ${ }^{17,18)}$ We suggest that changes in glial cell function, irrational ratio to neuron might be contributing to neurodevelopmental disorders. There is a possibility that changes in astrocyte generation might intervene with VPA teratogenic actions, leading to sustained, long-term, developmental effects on cortical function.

Previous studies provided the evidence that early VPA exposure alters cortical gliogenesis during a precarious stage of neurodevelopmental arrangement (PND 2 to 10). In our study we also suggest that altered of gliogenesis has a potential role in the impairment of neurobiological dysfunction in the frontal brain region that may induce the risk of behavioral-based neuropsychiatric disorders.

\section{acknowledgments}

The study was supported by 2015 Research Grant from Kangwon National University.

\section{REFERENCES}

1. Nedergaard M. Direct signaling from astrocytes to neurons in cultures of mammalian brain cells. Science 1994;263:17681771.

2. Mony TJ, Lee JW, Dreyfus C, DiCicco-Bloom E, Lee HJ. Valproic acid exposure during early postnatal gliogenesis leads to autistic-like behaviors in rats. Clin Psychopharmacol Neurosci 2016;14:338-344.

3. Kornack DR, Rakic P. Cell proliferation without neurogenesis in adult primate neocortex. Science 2001;294:2127-2130.

4. Rajkowska G, Miguel-Hidalgo JJ. Gliogenesis and g/ial pathology in depression. CNS Neurol Disord Drug Targets 2007; 6:219-233.

5. Lee HJ, Dreyfus C, DiCicco-Bloom E. Valproic acid stimulates proliferation of glial precursors during cortical gliogenesis in developing rat. Dev Neurobiol 2016;76:780-798.

6. Fatemi SH, Folsom TD, Reutiman TJ, Lee S. Expression of as- 
trocytic markers aquaporin 4 and connexin 43 is altered in brains of subjects with autism. Synapse 2008;62:501-507.

7. Laurence JA, Fatemi SH. Glial fibrillary acidic protein is elevated in superior frontal, parietal and cerebellar cortices of autistic subjects. Cerebellum 2005:4:206-210.

8. Bennett MR. Synapse formation and regression in the cortex during adolescence and in schizophrenia. Med J Aust 2009; 190(4 Suppl):S14-S16.

9. Sloan SA, Barres BA. Mechanisms of astrocyte development and their contributions to neurodevelopmental disorders. Curr Opin Neurobiol 2014;27:75-81.

10. Clarke LE, Barres BA. Emerging roles of astrocytes in neural circuit development. Nat Rev Neurosci 2013;14:311-321.

11. Auerbach BD, Osterweil EK, Bear MF. Mutations causing syndromic autism define an axis of synaptic pathophysiology. Nature 2011;480:63-68.

12. Berkel S, Marshall CR, Weiss B, Howe J, Roeth R, Moog U, et al. Mutations in the SHANK2 synaptic scaffolding gene in autism spectrum disorder and mental retardation. Nat Genet 2010:42:489-491.
13. Tidyman WE, Rauen KA. The RASopathies: developmental syndromes of Ras/MAPK pathway dysregulation. Curr Opin Genet Dev 2009;19:230-236.

14. Jacobs S, Nathwani M, Doering LC. Fragile X astrocytes induce developmental delays in dendrite maturation and synaptic protein expression. BMC Neurosci 2010;11:132.

15. Kim KC, Lee DK, Go HS, Kim P, Choi CS, Kim JW, et al. Pax6-dependent cortical glutamatergic neuronal differentiation regulates autism-like behavior in prenatally valproic acid-exposed rat offspring. Mol Neurobiol 2014;49: 512-528.

16. Kumamaru E, Egashira Y, Takenaka R, Takamori S. Valproic acid selectively suppresses the formation of inhibitory synapses in cultured cortical neurons. Neurosci Lett 2014;569: 142-147.

17. Bicks LK, Koike H, Akbarian S, Morishita H. Prefrontal cortex and social cognition in mouse and man. Front Psychol 2015;6:1805.

18. Lewis DA, Hashimoto T, Volk DW. Cortical inhibitory neurons and schizophrenia. Nat Rev Neurosci 2005;6:312-324. 